hep-ph $/ 9303317$

CERN-TH.6833/93

IEM-FT-69/93

\title{
On the electroweak phase transition in the Minimal Supersymmetric Standard Model
}

\author{
J.R. Espinosa \&, M. Quirós Đ \\ Instituto de Estructura de la Materia, CSIC \\ Serrano 123, E-28006 Madrid, Spain \\ and \\ F. Zwirner 3 \\ Theory Division, CERN \\ CH-1211 Geneva 23, Switzerland
}

\begin{abstract}
We study the finite-temperature effective potential of the Minimal Supersymmetric Standard Model, in the limit of only one light Higgs boson. Because of the large top Yukawa coupling, there can be significant differences with respect to the Standard Model case: for given values of the Higgs and top masses, little supersymmetry breaking in the stop sector can make the phase transition more strongly first-order. After including the full structure of the stop mass matrix, the most important experimental constraints and the leading plasma effects, we find that the present limits on Higgs and squark masses are still compatible with the scenario of electroweak baryogenesis, in a small region of parameter space corresponding to $m_{h} \lesssim 70 \mathrm{GeV}$ and $m_{\tilde{b}_{1}} \lesssim 105 \mathrm{GeV}$.
\end{abstract}

CERN-TH.6833/93

March 1993

\footnotetext{
${ }^{1}$ Supported by a grant of Comunidad de Madrid, Spain.

${ }^{2}$ Work partly supported by CICYT, Spain, under contract AEN90-0139.

${ }^{3}$ On leave from INFN, Sezione di Padova, Padua, Italy.
} 
1. The possibility of generating the cosmological matter-antimatter asymmetry at the electroweak phase transition has been the subject of lively discussions in the recent literature (for updated reviews and bibliography, see ref. [1]). Many difficult problems must be faced by any attempt at a quantitative model of electroweak baryogenesis, some of which are still awaiting definitive answers. Nevertheless, it is well established that the transition must be rather strongly first-order to avoid washing out any previously generated asymmetry円. As an approximate bound for the single-Higgs case one can take [2]

$$
\frac{v\left(T_{c}\right)}{T_{c}} \gtrsim 1
$$

where $T_{c}$ is the temperature at which the symmetric minimum is degenerate with the symmetrybreaking one, characterized by a vacuum expectation value $v\left(T_{c}\right)$. In the Standard Model (SM), this condition turns out to be incompatible with the experimental limit [3] on the Higgs mass,

$$
m_{h}>60 \mathrm{GeV} \quad(95 \% \text { c.l. }),
$$

even after implementing the presently known techniques [4,5] for handling the infrared problem. Simple extensions of the Standard Model, however, could still be acceptable candidates for electroweak baryogenesis [6]. Among them, a particularly attractive one is the Minimal Supersymmetric extension of the Standard Model (MSSM). In the MSSM (for a recent review see ref. [7]) there can be extra CP-violating phases [8] besides the Kobayashi-Maskawa one, which could help in generating the observed baryon asymmetry [9]. In the following, we shall deal with the effective potential of the MSSM (for simplicity, in the limit $m_{A} \rightarrow \infty$ with $\tan \beta$ fixed, corresponding to only one light Higgs with SM-like properties), to check whether conditions (11) and (2) can be simultaneously satisfied for some acceptable values of the parameters. Only in that case can there be room for some particular dynamical mechanism to work. Previous investigations have been carried out in ref. [10], using the $T$-dependent one-loop potential and assuming a universal soft supersymmetry-breaking mass for all squarks (other, more modeldependent considerations have been made in ref. [11]). In this paper, we improve over the previous analyses in two respects. First, we account for the full structure of the top-squark sector, which, because of the large top Yukawa coupling, is the only possible source of important deviations from the SM case, and we discuss the most important phenomenological constraints on the stop parameters. Second, we resum the leading plasma corrections to gauge and scalar boson masses, taking into account the enlarged particle spectrum of the MSSM: we compute additional contributions to vector bosons self-energies, as well as entirely novel effects associated with stop squarks self-energies.

The MSSM contains two complex Higgs doublets, $H_{1} \equiv\left(\begin{array}{c}H_{1}^{0} \\ H_{1}^{-}\end{array}\right)$and $H_{2} \equiv\left(\begin{array}{c}H_{2}^{+} \\ H_{2}^{0}\end{array}\right)$, and its tree-level potential reads, in standard notation [7]

$$
\begin{aligned}
V_{0} & =m_{1}^{2}\left|H_{1}\right|^{2}+m_{2}^{2}\left|H_{2}\right|^{2}+m_{3}^{2}\left(H_{1} H_{2}+\text { h.c. }\right) \\
& +\frac{g^{2}}{8}\left(H_{2}^{\dagger} \vec{\sigma} H_{2}+H_{1}^{\dagger} \vec{\sigma} H_{1}\right)^{2}+\frac{g^{\prime 2}}{8}\left(\left|H_{2}\right|^{2}-\left|H_{1}\right|^{2}\right)^{2} .
\end{aligned}
$$

\footnotetext{
${ }^{1}$ We are not considering here the alternative possibility of a net $(B-L)$ generated before the electroweak phase transition.

${ }^{2}$ One cannot rigorously exclude that large non-perturbative effects, not accounted for by the existing calculations, could modify the predicted values of the sphaleron energy and of $v\left(T_{c}\right) / T_{c}$ : we have nothing new to say in this respect, and in the following we shall disregard this possibility.
} 
It is not restrictive to assume that the only non-vanishing vacuum expectation values are $v_{1} \equiv$ $\left\langle H_{1}^{0}\right\rangle$ and $v_{2} \equiv\left\langle H_{2}^{0}\right\rangle$, both real and positive. Then, at the classical level, all Higgs masses and couplings can be expressed in terms of $\tan \beta \equiv v_{2} / v_{1}$ and $m_{A}=\left|m_{3}\right| \sqrt{\tan \beta+\cot \beta}$. Zerotemperature quantum corrections can be easily handled in full generality. At finite temperature, however, the analysis becomes much more complicated. Besides the obvious difficulty of studying a $T$-dependent potential in two variables, one might envisage the possibility of different nontrivial minima: some of them could break CP [12] or other symmetries of the MSSM, and it is not inconceivable that the phase transition might take place in two or more steps [13]. To simplify the problem, we consider here the limit $m_{A} \rightarrow \infty$, with $\tan \beta$ fixed. In this limit the low-energy theory contains the single Higgs doublet

$$
\Phi \equiv \cos \beta \bar{H}_{1}+\sin \beta H_{2}, \quad\left(\bar{H}_{1} \equiv-i \sigma^{2} H_{1}^{*}\right)
$$

with a tree-level potential of SM form, but with a special value of the quartic coupling. Calling $\phi / \sqrt{2}$ the constant background value of the real neutral component of $\Phi$, and restricting our attention to the $\phi$-dependence, we can write

$$
V_{0}=-\frac{\mu^{2}}{2} \phi^{2}+\frac{\lambda}{4} \phi^{4}
$$

with

$$
\lambda=\frac{g^{2}+g^{\prime 2}}{8} \cos ^{2} 2 \beta .
$$

In this case, the physical Higgs boson has couplings to vector bosons and fermions of SM strength, and in first approximation the limit of eq. (2) still applies. In general, $m_{3}^{2}$ is an independent, soft supersymmetry-breaking parameter, so that one can formally take the limit $m_{A} \rightarrow \infty$ while keeping all the supersymmetric particle masses finite. To decide whether this approximation is physically justified, one would need to know about spontaneous supersymmetry breaking in the underlying fundamental theory.

2. It is already clear from previous studies [10] that the only numerically relevant contributions to the one-loop finite-temperature effective potential of the MSSM (at the level of precision required by the problem under consideration) are, besides the SM ones, those associated with the stop squarks. It is then important to identify the region of the top-stop parameter space that is already ruled out by experiment. In the conservative limit of negligible mixing (as we shall see later, this is the most favourable situation for baryogenesis), the field-dependent stop and sbottom masses are given by

$$
\begin{array}{ll}
m_{\tilde{t}_{L}}^{2}(\phi)=m_{t}^{2}(\phi)+m_{Q_{3}}^{2}+D_{\tilde{t}_{L}}^{2}(\phi), & m_{\tilde{t}_{R}}^{2}(\phi)=m_{t}^{2}(\phi)+m_{U_{3}}^{2}+D_{\tilde{t}_{R}}^{2}(\phi), \\
m_{\tilde{b}_{L}}^{2}(\phi)=m_{b}^{2}(\phi)+m_{Q_{3}}^{2}+D_{\tilde{b}_{L}}^{2}(\phi), & m_{\tilde{b}_{R}}^{2}(\phi)=m_{b}^{2}(\phi)+m_{D_{3}}^{2}+D_{\tilde{b}_{R}}^{2}(\phi),
\end{array}
$$

where $m_{Q_{3}}, m_{U_{3}}$ and $m_{D_{3}}$ are soft supersymmetry-breaking parameters for $\left(\tilde{t}_{L}, \tilde{b}_{L}\right), \tilde{t}_{R}$ and $\tilde{b}_{R}$, respectively, and $(q=t, b)$

$$
D_{\tilde{q}_{L}}^{2}(\phi)=m_{Z}^{2}(\phi) \cos 2 \beta\left[T_{3 L}(\tilde{q})-Q(\tilde{q}) \sin ^{2} \theta_{W}\right], \quad D_{\tilde{q}_{R}}^{2}(\phi)=m_{Z}^{2}(\phi) \cos 2 \beta\left[Q(\tilde{q}) \sin ^{2} \theta_{W}\right] .
$$


The field-dependent masses for the gauge bosons and the third-generation quarks are given by

$$
m_{W}^{2}(\phi)=\frac{g^{2}}{4} \phi^{2}, \quad m_{Z}^{2}(\phi)=\frac{g^{2}+g^{2}}{4} \phi^{2}
$$

and

$$
m_{t}^{2}(\phi)=\frac{h_{t}^{2} \sin ^{2} \beta}{2} \phi^{2}, \quad m_{b}^{2}(\phi)=\frac{h_{b}^{2} \cos ^{2} \beta}{2} \phi^{2},
$$

where $h_{t}$ and $h_{b}$ are the top and bottom Yukawa couplings to $H_{2}^{0}$ and $H_{1}^{0}$, respectively.

To discuss the experimental constraints, it is appropriate to treat $m_{Q_{3}}, m_{U_{3}}$ and $m_{D_{3}}$ as independent parameters, unrelated to the soft supersymmetry-breaking masses of the first two generations. Specific models for the spontaneous breaking of local supersymmetry can predict some correlations among $m_{Q_{3}}, m_{U_{3}}, m_{D_{3}}$ and the other parameters of the MSSM, but we would like here to be as general as possible. Due to the smallness of $m_{b}$, bottom and sbottom loops do not play any important role in the description of the electroweak phase transition, so that we only need to consider the constraints on $m_{Q_{3}}$ and $m_{U_{3}}$. Nevertheless, $S U(2)_{L}$-invariance implies the equality of the soft masses for $\tilde{t}_{L}$ and $\tilde{b}_{L}$ : a strong constraint on their mass then comes from the fact that the decay $Z \rightarrow \tilde{b}_{L} \tilde{\bar{b}}_{L}$ has not been observed at LEP, either directly or via its contribution to the $Z$-boson width. Since the $Z \tilde{b}_{L} \tilde{\bar{b}}_{L}$ coupling is not particularly suppressed, being proportional to $\left|-(1 / 2)+(1 / 3) \sin ^{2} \theta_{W}\right| \simeq 0.42$, we can infer $m_{\tilde{b}_{L}} \gtrsim 45 \mathrm{GeV}$. Such a result translates into an excluded region in the $\left(m_{Q_{3}}, \tan \beta\right)$-plane, the one to the left of the solid line in fig. 1a: contours corresponding to higher values of $m_{\tilde{b}_{L}}$ are denoted by dashed lines. Direct squark searches at hadron colliders [15] do not provide model-independent limits on the squark masses of the third generation. First of all, the published limits assume five or six degenerate squark flavours. Secondly, even under such an assumption, the limit on the squark mass evaporates for a sufficiently large mass of the gluino or of the lightest supersymmetric particle. Other, indirect limits on $m_{Q_{3}}$ come from electroweak precision measurements, since the $\tilde{t}_{L}-\tilde{b}_{L}$ mass splitting can contribute significantly to the $\rho$ parameter [16],

$$
\Delta \rho(\tilde{t}, \tilde{b})=\frac{3 g^{2}}{64 \pi^{2} m_{W}^{2}}\left(m_{\tilde{t}_{L}}^{2}+m_{\tilde{b}_{L}}^{2}-2 \frac{m_{\tilde{t}_{L}}^{2} m_{\tilde{b}_{L}}^{2}}{m_{\tilde{t}_{L}}^{2}-m_{\tilde{b}_{L}}^{2}} \log \frac{m_{\tilde{t}_{L}}^{2}}{m_{\tilde{b}_{L}}^{2}}\right) .
$$

In the SM, the leading contribution to $\Delta \rho$ comes from the top-bottom mass splitting,

$$
\Delta \rho(t, b)=\frac{3 g^{2}}{64 \pi^{2} m_{W}^{2}}\left(m_{t}^{2}+m_{b}^{2}-2 \frac{m_{t}^{2} m_{b}^{2}}{m_{t}^{2}-m_{b}^{2}} \log \frac{m_{t}^{2}}{m_{b}^{2}}\right),
$$

but there are other contributions proportional to $\log \left(m_{h} / m_{Z}\right)$. In the case of a Higgs boson relatively close in mass to the $Z$ boson, as the one we are considering here, the latter contributions do not play an important role, and the bound from precision electroweak data [17] can be taken to be $\Delta \rho(t, b)<0.008$, corresponding to $m_{t}<160 \mathrm{GeV}$. In the MSSM, there are two types of contributions to $\Delta \rho$ (defined in terms of LEP observables) that can give measurable effects: The first type is associated with the vector boson self-energies at zero momentum, and includes the terms in eqs. (11) and (12). The second type is associated with the $Z$-boson selfenergy at $Q^{2} \simeq m_{Z}^{2}$ : in the presence of supersymmetric particles (e.g. charginos) slightly above

\footnotetext{
${ }^{3}$ Indeed, a refined analysis of the indirect effects of relatively light squarks should involve a full one-loop calculation of the precisely measured electroweak observables. However, this is beyond the aim of the present paper.
} 
threshold, there can be large effects [18 that compensate the zero-momentum contribution. Such cancellation, however, is only effective when considering LEP observables, but does not play a role in the fits to low-energy data. To take this possibility into account, we shall impose in the following the conservative bound $\Delta \rho(t, b)+\Delta \rho(\tilde{t}, \tilde{b})<0.01$. When the stop-sbottom contribution is negligible, this corresponds to $m_{t}<180 \mathrm{GeV}$. In the general case, this bound is represented by the solid lines in fig. 1b: for each indicated value of the top-quark mass, the region to the left of the corresponding line is excluded. For comparison, we also draw in fig. 1b dashed lines that correspond to the value $\Delta \rho(t, b)+\Delta \rho(\tilde{t}, \tilde{b})=0.008$. We are not aware of any model-independent bound on $m_{U_{3}}$.

3. We now move to the construction of the one-loop finite-temperature effective potential of the MSSM in the chosen limit. Keeping only the contributions associated with the gauge bosons $W$ and $Z$, with the quark $t$, and with the squark mass eigenstates $\tilde{t}_{1}$ and $\tilde{t}_{2}$, and working in the 't Hooft-Landau gauge and in the $\overline{D R}$-scheme, we can write

$$
V_{1}=V_{0}+\Delta V^{(0)}+\Delta V^{(T)}
$$

where $V_{0}$ has the form of eq. (5),

$$
\Delta V^{(0)}=\sum_{i=W, Z, t, \tilde{t}_{1}, \tilde{t}_{2}} \frac{n_{i}}{64 \pi^{2}} m_{i}^{4}(\phi)\left[\log \frac{m_{i}^{2}(\phi)}{Q^{2}}-\frac{3}{2}\right]
$$

and

$$
\Delta V^{(T)}=\frac{T^{4}}{2 \pi^{2}}\left[\sum_{i=W, Z, \tilde{t}_{1}, \tilde{t}_{2}} n_{i} J_{+}\left(y_{i}^{2}\right)+n_{t} J_{-}\left(y_{t}^{2}\right)\right]
$$

In eqs. (14) and (15),

$$
\begin{gathered}
n_{W}=6, \quad n_{Z}=3, \quad n_{t}=-12, \quad n_{\tilde{t}_{1}}=n_{\tilde{t}_{2}}=6, \\
y^{2} \equiv \frac{m^{2}(\phi)}{T^{2}}, \quad J_{ \pm}\left(y^{2}\right) \equiv \int_{0}^{\infty} d x x^{2} \log \left(1 \mp e^{-\sqrt{x^{2}+y^{2}}}\right),
\end{gathered}
$$

and $Q$ is the renormalization scale (we choose for definiteness $Q^{2}=m_{Z}^{2}$ ). For arbitrary mixing in the stop mass matrix the two field-dependent stop masses are given by

$$
m_{\tilde{t}_{1,2}}^{2}(\phi)=\frac{m_{\tilde{t}_{L}}^{2}(\phi)+m_{\tilde{t}_{R}}^{2}(\phi)}{2} \mp \sqrt{\left[\frac{m_{\tilde{t}_{L}}^{2}(\phi)-m_{\tilde{t}_{R}}^{2}(\phi)}{2}\right]^{2}+\frac{m_{t}^{2}(\phi) M_{t}^{2}}{\sin ^{2} \beta}}
$$

where $m_{\tilde{t}_{L}}^{2}(\phi)$ and $m_{\tilde{t}_{R}}^{2}(\phi)$ were given in eq. (17) and $M_{t} \equiv A_{t} \sin \beta+\mu \cos \beta$ is a mass parameter controlling the mixing in the stop mass matrixp.

In the chosen limit, the tree-level value of the Higgs mass is completely determined by $\tan \beta$, $m_{h}=m_{Z}|\cos 2 \beta|$, and the stop masses can be obtained from eq. (18) by replacing $\phi$ with its vacuum expectation value, $v \equiv\langle\phi\rangle$. To compute the radiatively corrected Higgs mass, and relate

\footnotetext{
${ }^{4}$ The phenomenological constraints on the top-bottom-stop-sbottom sector discussed before can be trivially generalized to the case of non-negligible mixing in the stop and sbottom mass matrices.

${ }^{5}$ In the general case, $M_{t}=0$ would imply $A_{t}=\mu=0$. In the case we are studying, however, $\tan \beta$ is not a dynamical variable: $M_{t}=0$ just defines the line $\mu=-A_{t} \tan \beta$ in the $\left(\mu, A_{t}\right)$ plane, and is consistent with $A_{t}, \mu \neq 0$.
} 
the renormalized parameter $\mu^{2}$ to physical quantities, one can easily adapt the calculations of refs. [14]. Defining $f\left(m^{2}\right) \equiv m^{4}\left[\log \left(m^{2} / Q^{2}\right)-(3 / 2)\right]$, one finds

$$
\mu^{2}=\frac{m_{Z}^{2} \cos ^{2} 2 \beta}{2}+\sum_{i} \frac{n_{i}}{64 \pi^{2}} \frac{1}{v} \frac{\partial m_{i}^{2}(v)}{\partial v} f^{\prime}\left[m_{i}^{2}(v)\right]
$$

and

$$
m_{h}^{2}=m_{Z}^{2} \cos ^{2} 2 \beta+\sum_{i} \frac{n_{i}}{64 \pi^{2}}\left\{\left[\frac{\partial m_{i}^{2}(v)}{\partial v}\right]^{2} f^{\prime \prime}\left[m_{i}^{2}(v)\right]+\left[\frac{\partial^{2} m_{i}^{2}(v)}{\partial v^{2}}-\frac{1}{v} \frac{\partial m_{i}^{2}(v)}{\partial v}\right] f^{\prime}\left[m_{i}^{2}(v)\right]\right\} .
$$

We are now ready to study the phase transition in the one-loop approximation. For simplicity, we begin by choosing a universal soft mass, $m_{Q_{3}}=m_{U_{3}} \equiv \tilde{m}$, and negligible mixing, $M_{t}=0$, in the stop mass matrix. To illustrate the effects of the radiative corrections to the Higgs mass, we plot, as dashed lines in fig. $2 \mathrm{a}$, contours of constant $m_{h}$ (in $\left.\mathrm{GeV}\right)$ in the $(\tilde{m}, \tan \beta)$ plane, for $m_{t}=150 \mathrm{GeV}$. The pattern of variation of the stop masses is not particularly interesting: within the intervals of $\tilde{m}$ and $\tan \beta$ considered in fig. $2 \mathrm{a}$, one finds $140 \mathrm{GeV} \lesssim m_{\tilde{t}_{1}} \lesssim 250 \mathrm{GeV}$, $145 \mathrm{GeV} \lesssim m_{\tilde{t}_{2}} \lesssim 250 \mathrm{GeV}$. As for the phase transition, we display as solid lines in fig. $2 \mathrm{a}$ contours of constant $v\left(T_{c}\right) / T_{c}$, obtained from the potential of eq. (13). We can see that the constraints (11) and (2) are satisfied in this case for $\tilde{m} \lesssim 120 \mathrm{GeV}$ and $\tan \beta \gtrsim 2.1$. On the other hand, after imposing the further constraints of fig. 1 we can see that the above bounds do not change appreciably, but the allowed region gets somewhat reduced. Imposing the more stringent constraint $\Delta \rho(t, b)+\Delta \rho(\tilde{t}, \tilde{b})<0.008$ would leave no acceptable region. At this level of approximation, the only difference with the SM case is the inclusion of the stop contributions to the one-loop effective potential. With those contributions removed, one would find for example that $m_{h}>60 \mathrm{GeV}$ implies $v\left(T_{c}\right) / T_{c} \lesssim 0.6$.

4. It is well known from SM studies that, to obtain a better estimate of $v\left(T_{c}\right) / T_{c}$ for the presently allowed values of the Higgs mass, it is of crucial importance to resum at least the leading infrared-dominated higher-loop contributions to the $T$-dependent effective potential, associated with the so-called daisy diagrams [4.5. In practice, this amounts to computing some $T$-dependent effective masses, $\bar{m}_{i}^{2}(\phi, T)$, for the light bosons of the model under consideration, and to correct the potential of eq. (13) with the addition of

$$
\Delta V_{\text {daisy }}=-\frac{T}{12 \pi} \sum_{i} n_{i}\left[\bar{m}_{i}^{3}(\phi, T)-m_{i}^{3}(\phi)\right] .
$$

The sum in eq. (21) should run over all the bosons of the model whose field-dependent masses in the relevant range of $\phi$ values are not significantly greater than $T_{c}$. Moreover, one must consider separately the transverse and longitudinal degrees of freedom of the gauge bosons, since their propagators are subject to different $T$-dependent corrections. In the SM, the most important effect is the Debye screening of the longitudinal gauge bosons. The inclusion of this screening [5] further reduces the value of $v\left(T_{c}\right) / T_{c}$. For example, in the SM with $m_{t}=150 \mathrm{GeV}$ and $m_{h}>60 \mathrm{GeV}$, the daisy-improved effective potential would give $v\left(T_{c}\right) / T_{c} \lesssim 0.45$.

Similar considerations can be made for the MSSM, but with some important differences. First of all, loops of supersymmetric particles can give additional contributions to the effective gauge boson masses. Secondly, when $m_{Q_{3}}, m_{U_{3}} \ll T$ the perturbative expansion in the stop 
squark sector, which is controlled by the parameters

$$
\alpha_{\tilde{t}_{1}, \tilde{t}_{2}}^{a} \equiv \frac{g_{a}^{2}}{2 \pi} \frac{T^{2}}{m_{\tilde{t}_{1}, \tilde{t}_{2}}^{2}}, \quad\left(g_{a}=g, g^{\prime}, g_{s}, h_{t}\right)
$$

breaks down at $\phi$ values close to 0 . Including the daisy diagrams amounts to a resummation to all orders in $\alpha_{\tilde{t}_{1}, \tilde{t}_{2}}^{a}$, and the Debye screening restores the validity of the stop perturbative expansion. When $m_{Q_{3}}, m_{U_{3}} \gg T$ the perturbative expansion in the stop sector is valid for all values of $\phi$. In that case the daisy resummation is unnecessary but harmless, since the relative contribution to (15) and (21) from $\tilde{t}_{1}$ and $\tilde{t}_{2}$ drops to zero. In the region $m_{Q_{3}}, m_{U_{3}} \sim T$, the screening from $m_{Q_{3}}, m_{U_{3}}$ competes with the Debye screening, which has to be taken into account. We then conclude that improving the theory by the daisy resummation is a consistent procedure 5 for the whole range of values of $m_{Q_{3}}, m_{U_{3}}$, and one has to compute the $T$-dependent self-energies for the stop sector, which involve gluon loops as well as loops of supersymmetric particles.

The masses $\bar{m}_{i}^{2}$ in (21) are given by

$$
\begin{gathered}
\bar{m}_{W_{T}}^{2}=m_{W}^{2}(\phi), \quad \bar{m}_{W_{L}}^{2}=m_{W}^{2}(\phi)+\Pi_{W_{L}}, \\
\bar{m}_{Z_{T}}^{2}=m_{Z}^{2}(\phi), \quad \bar{m}_{\gamma_{T}}^{2}=0, \\
\left(\begin{array}{cc}
\bar{m}_{Z_{L}}^{2} & 0 \\
0 & \bar{m}_{\gamma_{L}}^{2}
\end{array}\right)=R_{V}\left(\begin{array}{cc}
\frac{1}{4} g^{2} \phi^{2}+\Pi_{W_{L}} & -\frac{1}{4} g g^{\prime} \phi^{2} \\
-\frac{1}{4} g g^{\prime} \phi^{2} & \frac{1}{4} g^{\prime 2} \phi^{2}+\Pi_{B_{L}}
\end{array}\right) R_{V}^{-1}, \\
\bar{m}_{h}^{2}=\left(3 \lambda \phi^{2}-\mu^{2}\right)+\Pi_{h}, \quad \bar{m}_{\chi}^{2}=\left(\lambda \phi^{2}-\mu^{2}\right)+\Pi_{\chi}, \\
\left(\begin{array}{cc}
\bar{m}_{\tilde{t}_{1}}^{2} & 0 \\
0 & \bar{m}_{\tilde{t}_{2}}^{2}
\end{array}\right)=R_{\tilde{t}}\left(\begin{array}{cc}
\bar{m}_{\tilde{t}_{L}}^{2}(\phi)+\Pi_{\tilde{t}_{L}} & h_{t} M_{t} \phi \\
h_{t} M_{t} \phi & \bar{m}_{\tilde{t}_{R}}^{2}(\phi)+\Pi_{\tilde{t}_{R}}
\end{array}\right) R_{\tilde{t}}^{-1},
\end{gathered}
$$

where we have also included the Higgs $\left(n_{h}=1\right)$ and Goldstone $\left(n_{\chi}=3\right)$ degrees of freedom, and the rotations $R_{V}$ and $R_{\tilde{t}}$, which diagonalize the squared mass matrices for the neutral vector bosons and the stop squarks, respectively. The symbols $\Pi_{i}$ denote the (leading) parts of the $T$-dependent self-energies for the $i^{\text {th }}$ boson proportional to $T^{2}$. They are given by

$$
\begin{gathered}
\Pi_{W_{L}}=\frac{9}{4} g^{2} T^{2}+\frac{1}{12} g^{2}\left(\cos ^{4} \beta+\sin ^{4} \beta\right) T^{2}+\left\{2 g^{2} T^{2}\right\} \\
\Pi_{B_{L}}=\frac{85}{36} g^{\prime 2} T^{2}+\frac{1}{12} g^{\prime 2}\left(\cos ^{4} \beta+\sin ^{4} \beta\right) T^{2}+\left\{\frac{26}{9} g^{\prime 2} T^{2}\right\} \\
\Pi_{h}=\Pi_{\chi}=\frac{1}{24}\left[\frac{3}{4}\left(g^{2}+g^{\prime 2}\right)\left(\cos ^{4} \beta+\sin ^{4} \beta\right)-2 g^{\prime 2} \sin ^{2} \beta \cos ^{2} \beta\right] T^{2} \\
+\frac{3}{16} g^{2} T^{2}+\frac{1}{16} g^{\prime 2} T^{2}+\frac{3}{4} h_{t}^{2} \sin ^{2} \beta T^{2}+\left\{\frac{1}{8} g^{2} T^{2}+\frac{1}{24} g^{\prime 2} T^{2}\right\} \\
\Pi_{\tilde{t}_{L}}=\frac{4}{9} g_{s}^{2} T^{2}+\frac{1}{4} g^{2} T^{2}+\frac{1}{108} g^{\prime 2} T^{2}+\frac{1}{12} h_{t}^{2}\left(2+\sin ^{2} \beta\right) T^{2} \\
+\left\{\frac{2}{9} g_{s}^{2} T^{2}+\frac{1}{8} g^{2} T^{2}+\frac{1}{216} g^{\prime 2} T^{2}\right\}
\end{gathered}
$$

\footnotetext{
${ }^{6}$ In this paper we do not consider subleading corrections to the daisy approximation, which are controlled by the parameters $\beta_{\tilde{t}_{1}, \tilde{t}_{2}}^{a}=\left(g_{a}^{2} / 2 \pi\right)\left(T / m_{\tilde{t}_{1}, \tilde{t}_{2}}\right)$.
} 


$$
\Pi_{\tilde{t}_{R}}=\frac{4}{9} g_{s}^{2} T^{2}+\frac{4}{27} g^{\prime 2} T^{2}+\frac{1}{6} h_{t}^{2}\left(2+\sin ^{2} \beta\right) T^{2}+\left\{\frac{2}{9} g_{s}^{2} T^{2}+\frac{2}{27} g^{\prime 2} T^{2}\right\} .
$$

The previous expressions correspond to maximal screening, i.e. to the case where all supersymmetric particles (except the Higgs bosons which become superheavy in the limit $m_{A} \rightarrow \infty$ ) have masses smaller than or of the order of the critical temperature, and thus contribute to the selfenergies. Terms outside curly brackets correspond to the contribution from the SM particles and the squarks of the third generation. Terms in curly brackets correspond to the contribution from supersymmetric particles other than the squarks of the third generation, i.e. sleptons, squarks of the first and second generations, charginos, neutralinos and gluinos. The self-energies for the case of minimal screening, i.e. when the only light supersymmetric particles are the squarks of the third generation, can be read off (28)-(32) by removing the terms in curly brackets. Since gluinos provide the main contribution, $O\left(g_{s}^{2}\right)$, from the terms inside curly brackets, the cases of maximal and minimal screening can be thought of as cases of light and heavy gluinos. In particular, in the case of minimal screening the inclusion of light neutralinos, charginos, sleptons and squarks would not alter significantly the corresponding numerical results, and scenarios where the lightest supersymmetric particle is a light neutralino or a sneutrino are not excluded by our analysis.

The effects of including the resummation of the daisy diagrams, for the cases of maximal and minimal screening, are illustrated in figs. $2 \mathrm{~b}$ and $2 \mathrm{c}$, respectively. One can see that the allowed region can shrink considerably with respect to the one-loop approximation. In the case of maximal screening (which we use to emphasize the effect, but is not likely to correspond to a real situation) no acceptable points are left in the $(\tilde{m}, \tan \beta)$-plane. In the case of minimal screening, which should be interpreted as our best guess for a realistic situation, the acceptable region is reduced to a small triangle corresponding to $20 \mathrm{GeV} \lesssim \tilde{m} \lesssim 85 \mathrm{GeV}$ and $2 \lesssim \tan \beta \lesssim$ 2.8. Again, no acceptable region would be left if we had assumed the more stringent bound $\Delta \rho(t, b)+\Delta \rho(\tilde{t}, \tilde{b})<0.008$.

We now want to discuss the dependence of the results on the top-quark mass and on the stop mixing parameter, which is illustrated in figs. 3 and 4, respectively. In figs. 3a and 4a, solid lines correspond to the stop squark masses, the dashed lines to the Higgs mass, and the remaining free parameters are fixed to the representative values $\tilde{m}=50 \mathrm{GeV}, \tan \beta=2.25, M_{t}=0$ (fig. 3a) and $m_{t}=150 \mathrm{GeV}$ (fig. 4a). The dependences of $v\left(T_{c}\right) / T_{c}$ are illustrated in figs. $3 \mathrm{~b}$ and $4 \mathrm{~b}$ (solid lines), respectively: one can see that the most favourable situations are obtained for large top mass and small stop mixing. As for the effect of the top-quark mass, however, one should keep in mind the competing effect coming from the bound on $\Delta \rho$, which disfavours situations with large $m_{t}$ and small $\tilde{m}$. Using as before the conservative bound $\Delta \rho<0.01$, and working in the approximation of minimal screening, we obtain solutions for $130 \mathrm{GeV} \lesssim m_{t} \lesssim 160 \mathrm{GeV}$. Had we used the more stringent bound $\Delta \rho(t, b)+\Delta \rho(\tilde{t}, \tilde{b})<0.008$, we would have found solutions only for $130 \mathrm{GeV} \lesssim m_{t} \lesssim 140 \mathrm{GeV}$.

Before concluding we would like to discuss the uncertainty in the definition of the critical temperature $T_{c}$ and its influence on the calculation of $v\left(T_{c}\right) / T_{c}$. We have defined $T_{c}$ as the temperature at which the symmetric minimum is degenerate with the symmetry-breaking one. This definition does correspond to the onset of the phase transition, though the latter will take place at a somewhat lower temperature $T_{\mathrm{ph}}$. On the other hand, the end of the phase transition happens at a second critical temperature $T_{c}^{\prime}$, which is defined as the temperature at which the curvature of the effective potential vanishes at the origin. It is also clear that $T_{c}^{\prime} \lesssim T_{\mathrm{ph}}$, and so

$$
\frac{v\left(T_{c}\right)}{T_{c}} \lesssim \frac{v\left(T_{\mathrm{ph}}\right)}{T_{\mathrm{ph}}} \lesssim \frac{v\left(T_{c}^{\prime}\right)}{T_{c}^{\prime}} .
$$


We plot the values of $v\left(T_{c}^{\prime}\right) / T_{c}^{\prime}$ as dashed lines in figs. $3 \mathrm{~b}$ and $4 \mathrm{~b}$. The quantity $v\left(T_{\mathrm{ph}}\right) / T_{\mathrm{ph}}$ lies in the stripe between the solid and dashed lines. We have adopted in this paper the bound of eq. (1), which is consistent with the bound $v\left(T_{c}^{\prime}\right) / T_{c}^{\prime} \gtrsim 1.3$, which was used in some of the previous analyses [2, 2, 6, 10].

Finally, we need to discuss the effects of relaxing the assumption of a universal soft mass in the stop sector. In this case one can take advantage of the fact that $m_{U_{3}}$ can be pushed to very small values without violating any bound. The most favourable situation is the one with large $m_{Q_{3}}$, so that the contribution to $\Delta \rho$ is negligible, and very small $m_{U_{3}}$. One can then find solutions up to the value of $m_{t}$ that saturates the bound on $\Delta \rho$.

5. To summarize our results, the region of the MSSM parameter space that is still compatible with the scenario of electroweak baryogenesis and with the present experimental constraints is quite restricted. It seems unlikely that it can be matched with universal boundary conditions on soft supersymmetry-breaking masses at the grand-unification scale, as is often assumed by model builders. A thorough and reliable discussion of this point, however, would be rather complicated. Moreover, there is no strong theoretical argument to forbid deviations from universality, especially in the stop-sbottom sector. In the case of general supersymmetry breaking considered in this paper, the allowed region corresponds to a rather light spectrum in the Higgs and stop-sbottom sectors, $m_{h} \lesssim 70 \mathrm{GeV}$ and $m_{\tilde{b}_{1}} \lesssim 105 \mathrm{GeV}$ : these predictions should be definitely tested at LEP II in the forthcoming years. Our results are quite sensitive to the assumed bound on $\Delta \rho$ : as is clear from fig. $1 \mathrm{~b}$, more stringent bounds on $\Delta \rho$ drastically reduce the allowed region of parameter space. Finally, we would like to recall that our analysis was carried out in the limit of a single light Higgs: as already mentioned, the case of small $m_{A}$ is much more cumbersome to analyse and goes beyond the aim of the present paper.

\section{Acknowledgements}

We would like to thank R. Barbieri, F. Caravaglios and M.E. Shaposhnikov for useful discussions. 


\section{References}

1. M.E. Shaposhnikov, in 'Proceedings of the 1991 Summer School in High Energy Physics and Cosmology', Trieste, 17 June-9 August 1991, E. Gava et al. eds. (World Scientific, Singapore, 1992), Vol. 1, p. 338; A.G. Cohen, D.B. Kaplan and A.E. Nelson, San Diego preprint UCSD-PTH-93-02, BUHEP-93-4.

2. M.E. Shaposhnikov, JETP Lett. 44 (1986) 364; Nucl. Phys. B287 (1987) 757 and B299 (1988) 797; A.I. Bochkarev, S.Yu. Klebnikov and M.E. Shaposhnikov, Nucl. Phys. B329 (1990) 490; M. Dine, P. Huet and R. Singleton Jr., Nucl. Phys. B375 (1992) 625.

3. J.-F. Grivaz, Orsay preprint LAL 92-59, and references therein.

4. S. Weinberg, Phys. Rev. D9 (1974) 3357; L. Dolan and R. Jackiw, Phys. Rev. D9 (1974) 3320; D.A. Kirzhnits and A.D. Linde, Zh. Eksp. Teor. Fiz. 67 (1974) 1263 and Ann. Phys. 101 (1976) 195; P. Fendley, Phys. Lett. B196 (1987) 175.

5. M.E. Carrington, Phys. Rev. D45 (1992) 2933; M. Dine, R.G. Leigh, P. Huet, A. Linde and D. Linde, Phys. Lett. B283 (1992) 319 and Phys. Rev. D46 (1992) 550; P. Arnold, Phys. Rev. D46 (1992) 2628; J.R. Espinosa, M. Quirós and F. Zwirner, Phys. Lett. B291 (1992) 115 and preprint CERN-TH.6577/92, IEM-FT-58/92; C.G. Boyd, D.E. Brahm and S.D. Hsu, Caltech preprint CALT-68-1795, HUTP-92-A027, EFI-92-22; W. Buchmüller and T. Helbig, preprint DESY 92-117; W. Buchmüller, T. Helbig and D. Walliser, preprint DESY 92-151; P. Arnold and O. Espinosa, Univ. of Washington preprint UW/PT-92-18; W. Buchmüller, Z. Fodor, T. Helbig and D. Walliser, preprint DESY 93-021.

6. A.I. Bochkarev, S.V. Kuzmin and M.E. Shaposhnikov, Phys. Lett. B244 (1990) 275 and Phys. Rev. D43 (1991) 369; N. Turok and J. Zadrozny, Phys. Rev. Lett. 65 (1990) 2331, Nucl. Phys. B358 (1991) 471 and B369 (1992) 728; L. Mc Lerran, M. Shaposhnikov, N. Turok and M. Voloshin, Phys. Lett. B256 (1991) 451; B. Kastening, R.D. Peccei and X. Zhang, Phys. Lett. B266 (1991) 413; G.W. Anderson and L.J. Hall, Phys. Rev. D45 (1992) 2685; M. Dine, P. Huet, R. Singleton Jr. and L. Susskind, Phys. Lett. B257 (1991) 351; K. Enqvist, K. Kainulainen and I. Vilja, Phys. Rev. D45 (1992) 3415; N. Sei, I. Umemura and K. Yamamoto, Phys. Lett. B299 (1993) 286; M. Pietroni, Padova preprint DFPD/92/TH/36; J.R. Espinosa and M. Quirós, Madrid preprint IEM-FT-67/93, to appear in Phys. Lett. B.

7. F. Zwirner, in 'Proceedings of the 1991 Summer School in High Energy Physics and Cosmology', Trieste, 17 June-9 August 1991, E. Gava et al. eds. (World Scientific, Singapore, 1992), Vol. 1, p. 193.

8. J. Ellis, S. Ferrara and D.V. Nanopoulos, Phys. Lett. B114 (1982) 231; W. Buchmüller and D. Wyler, Phys. Lett. B121 (1983) 321; J. Polchinski and M.B. Wise, Phys. Lett. B125 (1983) 393; F. del Aguila, B. Gavela, J. Grifols and A. Mendez, Phys. Lett. B126 (1983) 71; D.V. Nanopoulos and M. Srednicki, Phys. Lett. B128 (1983) 61; M. Dugan, B. Grinstein and L.J. Hall, Nucl. Phys. B255 (1985) 413.

9. A.G. Cohen and A.E. Nelson, Phys. Lett. B297 (1992) 111.

10. G.F. Giudice, Phys. Rev. D45 (1992) 3177; S. Myint, Phys. Lett. B287 (1992) 325. 
11. D.V. Nanopoulos and H. Pois, preprint CERN-TH.6763/92.

12. D. Comelli and M. Pietroni, Padova preprint DFPD-93/TH/06, UTS-DFT-93-1.

13. D. Land and E.D. Carlson, Phys. Lett. B292 (1992) 107; E.D. Carlson and W.D. Garretson, Harvard preprint HUTP-92/A067.

14. Y. Okada, M. Yamaguchi and T. Yanagida, Prog. Theor. Phys. Lett. 85 (1991) 1; J. Ellis, G. Ridolfi and F. Zwirner, Phys. Lett. B257 (1991) 83; R. Barbieri and M. Frigeni, Phys. Lett. B258 (1991) 395; J. Ellis, G. Ridolfi and F. Zwirner, Phys. Lett. B262 (1991) 477.

15. F. Abe et al. (CDF Collaboration), Phys. Rev. Lett. 69 (1992) 3439.

16. L. Alvarez-Gaumé, J. Polchinski and M. Wise, Nucl. Phys. B221 (1983) 495; R. Barbieri and L. Maiani, Nucl. Phys. B224 (1983) 32; C.S. Lim, T. Inami and N. Sakai, Phys. Rev. D29 (1984) 1488.

17. L. Rolandi, preprint CERN-PPE-92-175, and references therein.

18. R. Barbieri, M. Frigeni and F. Caravaglios, Phys. Lett. B279 (1992) 169; J. Ellis, G.L. Fogli and E. Lisi, preprint CERN-TH.6643/92.

\section{Figure captions}

Fig.1: Exclusion contours in the $\left(m_{Q_{3}}, \tan \beta\right)$ plane, for negligible stop mixing, corresponding to different experimental constraints: a) the solid line corresponds to $m_{\tilde{b}_{L}}=45 \mathrm{GeV}$, the dashed lines to the indicated higher values of $m_{\tilde{b}_{L}}$ (in $\mathrm{GeV}$ ); b) for each indicated value of $m_{t}$ (in $\mathrm{GeV}$ ), the solid lines correspond to $\Delta \rho=0.01$, the dashed lines to $\Delta \rho=0.008$.

Fig.2: Lines of constant $v\left(T_{c}\right) / T_{c}$ (solid) and $m_{h}$ (dashed, in $\mathrm{GeV}$ ) in the $\left(m_{Q_{3}}, \tan \beta\right.$ ) plane, for $m_{Q_{3}}=m_{U_{3}} \equiv \tilde{m}, m_{t}=150 \mathrm{GeV}$ and $M_{t}=0$ : a) one-loop approximation; b) daisyimproved, for maximal screening; c) daisy-improved, for minimal screening.

Fig.3: Dependence on $m_{t}$ of some relevant quantities in the representative case $m_{Q_{3}}=m_{U_{3}} \equiv$ $\tilde{m}=50 \mathrm{GeV}, \tan \beta=2.25, M_{t}=0$ : a) Higgs (dashed line) and stop masses (solid line), in $\mathrm{GeV}$; b) $v\left(T_{c}\right) / T_{c}$ (solid line) and $v\left(T_{c}^{\prime}\right) / T_{c}^{\prime}$ (dashed line), daisy-improved for minimal screening.

Fig.4: Dependence on $M_{t}$ of some relevant quantities in the representative case $m_{t}=150 \mathrm{GeV}$, $m_{Q_{3}}=m_{U_{3}} \equiv \tilde{m}=50 \mathrm{GeV}, \tan \beta=2.25$ : a) Higgs (dashed line) and stop masses (solid line), in $\mathrm{GeV}$; b) $v\left(T_{c}\right) / T_{c}$ (solid line) and $v\left(T_{c}^{\prime}\right) / T_{c}^{\prime}$ (dashed line), daisy-improved for minimal screening. 